\author{
Diana Patricia Román Tobón ${ }^{1}$ \\ Alberto Rafael Vargas García2,* \\ 1. Enfermera Especialista en Cuidado al Adulto en Estado Crítico de Salud. Diplomada \\ en Cuidado de Enfermería en el Paciente con Enfermedad Vascular. Diplomada en \\ Atención de Enfermería a Pacientes con Heridas de lo Básico a lo Avanzado. Enfermera \\ de Clínica de Heridas. Clínica Cardio VID, Medellín, Colombia. \\ 2. Enfermero Magíster en Epidemiología. Diplomado en Epidemiología Clínica y Práctica \\ Basada en la Evidencia. Coordinador de Vigilancia Epidemiológica e Infección \\ Intrahospitalaria. Clínica Cardio VID, Medellín, Colombia. Docente hora cátedra \\ Universidad Pontificia Bolivariana.
}

*Autor para correspondencia.

Correo electrónico: avargasg@vid.org.co (Alberto Rafael Vargas García).

\section{Descripción de las lesiones relacionadas con la dependencia en una clínica cardiovascular} Dependence-related lesions
in a cardiovascular clinic

Recibido el 9 de diciembre de 2019; aceptado el 17 de marzo de 2020.

\section{RESUMEN}

Objetivos: Describir las lesiones relacionadas con la dependencia en una clínica cardiovascular de alto nivel de atención, de la Ciudad de Medellín, Colombia, en el año 2018. Metodología: Estudio descriptivo retrospectivo con una muestra no probabilística a conveniencia. A las variables de naturaleza cuantitativa, previa prueba de normalidad de Shapiro Wilk, se les calcularon la mediana con sus respectivos rangos intercuartílicos y los valores mínimo y máximo. Las variables de naturaleza cualitativa se presentan con frecuencias absolutas y relativas. Resultados: Se presentaron 167 pacientes con lesiones para una incidencia global del 2,8\%; las lesiones más frecuentes fueron causadas por dispositivos médicos (50,9\%). Las lesiones por apoyo fueron del 28,7\%; por adhesivo, del 18,6\%; y por humedad, del 1,8\%. No se presentaron lesiones por fricción. El 73,1\% de las lesiones se presentaron en pacientes adultos. El 77,2\% tenía clasificación de riesgo alto; el 60,5\% de las lesiones fueron de categoría 1 . De acuerdo con el sitio anatómico, la mayoría de las lesiones se presentaron en cara y cuello (47,9\%), seguido de pelvis y miembros inferiores (40,1\%). En el análisis de calidad se encontró que el 60,5\% fue evitable. Conclusiones: La mayoría de las lesiones relacionadas con la dependencia fueron secundarias

al uso de dispositivos médicos. Es necesario continuar haciendo estudios que brinden información sobre los diferentes tipos de lesiones por dependencia, ya que las medidas de prevención y los cuidados de estas varían de acuerdo con el mecanismo de producción.

PALABRAS CLAVE: Úlcera por presión, dispositivo médico, prevención y control, tejido adhesivo, herida y lesión.

\section{ABSTRACT}

Aim: To describe dependence-related lesions in a high level of care cardiovascular clinic in the city of Medellin, Colombia, in 2018. Method: Retrospective descriptive study with a non-probabilistic sample. Quantitative data were calculated with median and their respective interquartile ranges, minimum and maximum values. Qualitative data is presented with absolute and relative frequencies. Results: 167 patients suffered dependence-related lesions, incidence $2.8 \%$. The most frequent lesions were caused by medical devices (50.9\%), by pressure (28.7), by Medical Adhesive-Related Skin Injury (18.6), and by humidity (1.8\%). There were not friction injuries. $73.1 \%$ of the lesions occurred in adult patients. $77.2 \%$ had a high risk classification; $60.5 \%$ of the injuries were category 1 . According to the anatomical site, most lesions were presented in the face and neck (47.9\%), followed by the pelvis and lower limbs $(40.1 \%)$. In the quality analysis it was found that $60.5 \%$ were avoidable. Conclusions: The majority of dependence-related lesions were secondary to the use of medical devices. It is necessary to make more researches that show the different type of dependence-related lesions, since caring and prevention measures vary according to the production mechanism.

KEYWORDS: Pressure Ulcer, Equipment and supplies, prevention and control, Tissue Adhesives, wound and injury.

\section{У INTRODUCCIÓN}

Las lesiones por presión son eventos adversos que representan una carga agregada a la enfermedad y una menor calidad de vida para el paciente y para los que están a su cuidado, además de los costos en salud representados por el aumento en la estancia hospitalaria, tratamiento para el alivio del dolor o para el control de la infección, intervenciones quirúrgicas e insumos utilizados para la curación de las lesiones. Se debe considerar también que pueden generarse implicaciones legales, ya que en la mayoría de los casos son prevenibles y su prevención requiere un manejo integral e interdisciplinario en el abordaje del paciente $e^{1-4}$.
Tradicionalmente, todas las alteraciones que presentaban los pacientes en la piel eran consideradas como úlceras por presión (UPP); sin embargo, en años recientes, el Grupo Nacional para el Estudio y Asesoramiento en Úlceras por Presión y Heridas Crónicas (GNEAUPP) y el National Pressure Ulcer Advisory Panel (NPUAP) propusieron utilizar el término lesión en lugar de úlcera, debido a que puede haber un daño del tejido tegumentario sin que se presente alteración de la integridad cutánea, con lo cual se comienza a hablar de lesiones por presión (LPP). Así mismo, ya que no todas las lesiones son ocasionadas por la presión, García Fernández et al. proponen, según el mecanismo de producción, un nuevo término: lesiones asociadas a la dependencia, 
las cuales pueden ser clasificadas como: UPP y cizalla o combinación de ambas; úlceras por humedad; lesiones por fricción, y lesiones mixtas o combinadas 5 . Posteriormente, se comienza a hablar de lesiones relacionadas con adhesivos o MARSI (Medical Adhesive-Related Skin Injuries) por sus siglas en inglés ${ }^{6}$.

La UPP se define como: "Una lesión localizada en la piel y/o el tejido subyacente por lo general sobre una prominencia ósea, como resultado de la presión, o la presión en combinación con las fuerzas de cizalla. En ocasiones, también pueden aparecer sobre tejidos blandos sometidos a presión externa por diferentes materiales o dispositivos clínicos"5. Con base en esta definición se pueden diferenciar dos tipos de lesiones cuya causa es la presión: $a$ ) las que se presentan sobre prominencias óseas y que están relacionadas con las superficies donde se apoyan los pacientes al estar acostados o sentados, también llamadas lesiones por apoyo y $b$ ) las lesiones que están asociadas al uso de los dispositivos médicos al estar en contacto con la piel o las mucosas del paciente: lesiones por dispositivo (LPD).

La clasificación de las LPP se realiza de acuerdo con su gravedad y profundidad. En la categoría 1, la piel está intacta y presenta un enrojecimiento no blanqueable al retirar la presión, generalmente ubicada sobre una prominencia ósea. En la categoría 2 hay pérdida del espesor parcial de la dermis que se presenta como una lesión poco profunda y con un lecho rojo o rosado. En la categoría 3 hay pérdida total del grosor de la piel, la grasa subcutánea puede ser visible, pero los huesos, tendones o músculos no están expuestos. En la categoría 4 hay pérdida total del espesor del tejido con exposición de hueso, tendón o músculo. En la lesión no clasificable o de profundidad desconocida hay pérdida total del espesor de los tejidos en donde la base de la herida está cubierta por tejido de esfacelo y/o necrosis seca, y en la sospecha de lesión de tejidos profundos hay un área de piel púrpura, marrón o de decoloración intacta o ampolla (flictena) llena de sangre, producida por la lesión a tejidos subyacentes por la presión y/o cizalla ${ }^{7}$.

Las LPD se presentan porque el dispositivo por sí solo crea presión, lo que junto con la humedad y el calor que se desarrolla entre este y la piel cambian el microclima de la misma, lo que hace al paciente más susceptible de padecer una lesión. Además, estos dispositivos deben fijarse adecuadamente para evitar su salida accidental o para mantener un sellado adecuado, como es el caso de las máscaras de ventilación no invasiva, creando presión en sitios diferentes a las prominencias óseas. La lesión resultante de la presión por el dispositivo clínico se adapta al patrón o la forma del dispositivo ${ }^{8,9}$ (figs. 1 y 2).

Las lesiones por fricción (LPF) son lesiones localizadas en la piel, que generalmente no afecta a los tejidos subyacentes, provocadas por las fuerzas derivadas del roce-fricción entre la piel del paciente y otra superficie paralela, que al contacto con él se mueven en sentido contrario 5 .

Las lesiones cutáneas asociadas a la humedad (LESCAH) son lesiones que se presentan por la exposición prolongada a diversas fuentes de humedad como orina, heces, exudados de heridas, efluentes de estomas que generan inflamación y/o erosión de la piel ${ }^{5,10,11}$.

El daño de la piel por uso de adhesivo o MARSI es una lesión traumática que aparece como resultado de la ruptura de la epidermis y su separación de la dermis, debido a las fuerzas de tracción del adhesivo sobre la piel ocasionada por una inadecuada colocación y/o retirada del adhesivo, el cual puede ser una cinta, tejido poroso, apósito transparente o cualquier otro dispositivo que se fije a la piel por medio de adhesivo ${ }^{6}$. Esta anormalidad cutánea permanece 30 minutos o más después de retirar dicho adhesivo (fig. 3).

La epidemiología de las LPP es muy variable y difiere de una región a otra. En países europeos, la prevalencia se encuentra entre el 8,0\% y el 18,1\% ${ }^{12-14}$; en Estados Unidos es del 10,6\% $0^{15}$; en Latinoamérica reportan prevalencias hasta del $23,0 \%{ }^{16}$, y en Colombia se encuentran prevalencias desde el 5,6\% hasta el $31,2 \%$, siendo mayor en las unidades de cuidados intensivos ${ }^{17-21}$.
La literatura científica reporta que la prevalencia de lesiones secundarias a dispositivos médicos llega hasta el 25,0\% ${ }^{22-25}$. La incidencia de LESCAH es del 13,2\% y la de LPF del 15,8\% ${ }^{26}$. La incidencia acumulada de MARSI se reporta en un $15,5 \%$ en pacientes adultos mayores ${ }^{27}$.

Este estudio pretende describir las características de las lesiones relacionadas con la dependencia que presentaron los pacientes adultos y pediátricos atendidos en una institución cardiovascular de alta complejidad.

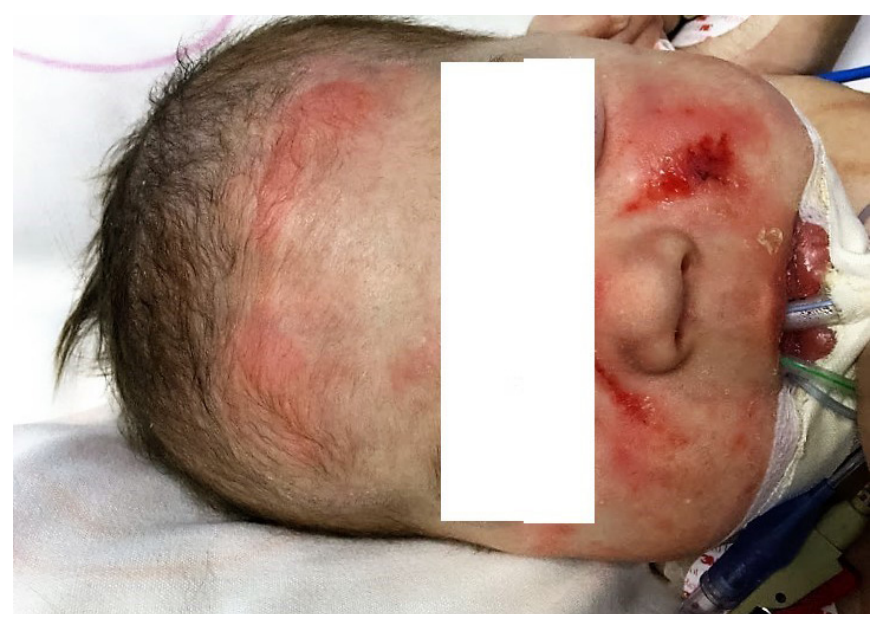

Figura 1. Lesión por dispositivo médico. Máscara de ventilación no invasiva. Tomada de: Base de datos de la Clínica de heridas. Cardio VID, 2018.

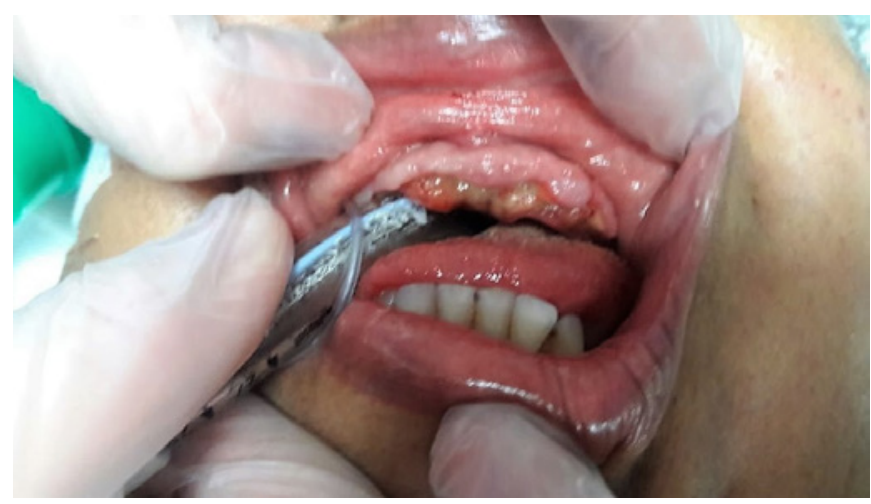

Figura 2. Lesión por dispositivo médico. Tubo orotraqueal. Tomada de: Base de datos de la Clínica de heridas. Cardio VID, 2018.

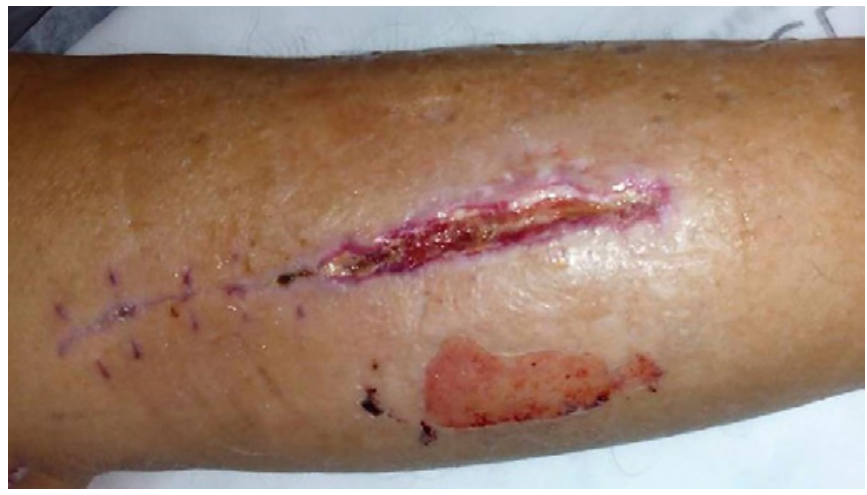

Figura 3. Lesión por adhesivo. MARSI.

Tomada de: Base de datos de la Clínica de heridas. Cardio VID, 2018 


\section{У METODOLOGÍA}

Estudio observacional descriptivo retrospectivo de enero a diciembre de 2018. La población fueron pacientes adultos, pediátricos y neonatos ingresados en la institución en este periodo. Los criterios de inclusión fueron: pacientes adultos, pediátricos y neonatos que presentaron lesiones por dependencia asociadas a la atención en salud. Los criterios de exclusión: pacientes que al momento del ingreso a la institución presentaban lesiones por dependencia, eventos adversos reportados como incidentes e historia clínica incompleta. Muestra no probabilista a conveniencia de 167 pacientes. Fuentes secundarias de historias clínicas, reporte de eventos adversos y base de datos de clínica de heridas. Los datos fueron recolectados por los investigadores principales, y para asegurar la calidad de la información fueron revisados con el fin de verificar datos faltantes o errores de digitación. La clasificación de la lesión relacionada con la dependencia fue realizada por la coordinadora de gestión de la seguridad del paciente y la coordinadora de la clínica de heridas, basada en las definiciones del GNEAUPP, con el fin de evitar el sesgo de selección. Las variables evaluadas fueron: tipo de paciente y edad; características de la lesión por: tipo, categoría, sitio anatómico, tiempo de aparición, servicio de origen, clasificación del riesgo de acuerdo con la escala Braden, Braden Q modificada

Tabla 1. Características de las lesiones por dependencia

\begin{tabular}{|c|c|c|c|}
\hline & Característica & $N=167$ & $(\%)$ \\
\hline \multirow{2}{*}{$\begin{array}{l}\text { Tipo de } \\
\text { paciente }\end{array}$} & Adulto & 122 & 73,1 \\
\hline & Pediátrico & 45 & 26,9 \\
\hline \multirow{5}{*}{ Edad } & Menor de 2 años & 38 & 22,8 \\
\hline & 2 a 17 años & 7 & 4,2 \\
\hline & 18 a 50 años & 15 & 9,0 \\
\hline & 51 a 65 años & 60 & 35,9 \\
\hline & $>66$ años & 47 & 28,1 \\
\hline \multirow{4}{*}{ Tipo de lesión } & Dispositivo & 85 & 50,9 \\
\hline & Por apoyo & 48 & 28,7 \\
\hline & MARSI & 31 & 18,6 \\
\hline & Humedad & 3 & 1,8 \\
\hline \multirow{7}{*}{$\begin{array}{l}\text { Servicio de } \\
\text { procedencia }\end{array}$} & UCla Adultos & 70 & 41,9 \\
\hline & Hospitalización adultos & 43 & 25,7 \\
\hline & UCI Cardiopediátrica & 37 & 22,2 \\
\hline & Cirugía & 8 & 4,8 \\
\hline & Urgencias & 5 & 3 \\
\hline & Hemodinámica & 2 & 1,2 \\
\hline & Hospitalización Pediatría & 2 & 1,2 \\
\hline \multirow{3}{*}{ Categoría } & 1 & 101 & 60,5 \\
\hline & 2 & 64 & 38,3 \\
\hline & 3 & 2 & 1,2 \\
\hline \multirow{4}{*}{$\begin{array}{l}\text { Clasificación } \\
\text { de riesgo }\end{array}$} & Alto & 129 & 77,2 \\
\hline & Bajo & 26 & 15,6 \\
\hline & Moderado & 9 & 5,4 \\
\hline & No clasificado & 3 & 1,8 \\
\hline \multirow{2}{*}{$\begin{array}{l}\text { Clasificación } \\
\text { del evento }\end{array}$} & Evitable & 101 & 60,5 \\
\hline & No evitable & 66 & 39,5 \\
\hline
\end{tabular}

anidad de Cuidados Intensivos

MARSI: Medical Adhesive-Related Skin Injuries; UCl: unidad de cuidados intensivos. y Neonatal Skin Risk Assessment Scale (NSRAS); clasificación del evento en evitable y no evitable como parámetro de calidad.

$\mathrm{El}$ análisis de la información se realizó con el paquete estadístico SPSS $^{\bullet}$ versión 21 (SPSS Inc.; Chicago, Illinois, USA). Las variables cualitativas se analizaron con frecuencias absolutas y relativas, y las cuantitativas, previa prueba de normalidad de Shapiro Wilk, con medidas de tendencia central, dispersión o de posición.

\section{$\searrow$ CONSIDERACIONES ÉTICAS}

De acuerdo con la Resolución 8430 de 1993 de la legislación colombiana, este es un estudio sin riesgo, ya que implementó técnicas y métodos de forma retrospectiva y no se realizó ninguna intervención o modificación intencionada de las variables biológicas, fisiológicas, psicológicas o sociales de los individuos de investigación. Los investigadores siguieron los lineamientos de la declaración de Helsinki actualizada de 2013.

La privacidad se conservó utilizando los números de historia clínica de los pacientes y no los nombres. Los datos han sido utilizados exclusivamente para fines investigativos y mejora de los procesos de atención y seguridad del paciente.

Se respetaron los principios éticos fundamentales y se solicitó autorización al Comité de ética en investigación de la institución para acceder a la información de los pacientes.

\section{$\vee$ RESULTADOS}

Se presentaron 167 lesiones por dependencia que representaron una tasa global del 2,8\%. Por servicios, la unidad de cuidados intensivos cardiopediátrica (UCICP) presentó una incidencia del 10,8\% y la unidad de cuidados intensivos adultos (UCIA), del 6,1\%. La mayoría de las lesiones se presentaron en pacientes mayores de 51 años; las lesiones más frecuentes fueron causadas por dispositivos médicos (50,9\%) (tabla 1).

De acuerdo con el sitio anatómico, la mayoría de las lesiones se presentaron en cara y cuello con un 47,9\%; de estas, los sitios más frecuentes fueron los labios $(10,8 \%)$ y la fosa nasal (10,2\%). El 40,1\% del total de lesiones se presentaron en la pelvis y miembros inferiores, siendo la región sacra $(13,2 \%)$, el meato urinario $(9,0 \%)$ y los glúteos $(8,4 \%)$ los sitios más recurrentes (tabla 2).

\section{$Y$ LESIONES POR DISPOSITIVOS MÉDICOS}

La mayoría de las lesiones $(85,50,9 \%)$ fueron ocasionadas por el uso de dispositivos médicos, de las cuales el 48,2\% se presentaron en UCIA, el 22,3\% en UCIP y el 21,1\% en el servicio de hospitalización adultos. En cuanto al compromiso, el 43,0\% fue de categoría 2 y el 57,0\% de categoría 1 . El $62,3 \%$ se presentó en pacientes mayores de 51 años y el $16,4 \%$ en pacientes entre 1 y 6 meses de edad. El 88,2\% de los pacientes tenía clasificación de riesgo alto.

El dispositivo que más generó lesiones fue el tubo orotraqueal con un $35,3 \%$. La incidencia de lesiones por este dispositivo fue del 4,3\% en adultos y del 3,3\% en pacientes pediátricos. De acuerdo con el sitio anatómico, el $63,3 \%$ se presentó en los labios, el 20,0\% en las encías y el $16,7 \%$ en el paladar. El $100 \%$ tuvo clasificación de riesgo alto.

La lesión por sonda vesical representó el $18,8 \%$; solo en adultos, la incidencia fue del 3,1\%. El 75,0\% se presentó en la UCIA. El 67,5\% 
Tabla 2. Sitio anatómico donde se presentaron las úlceras por dependencia

\begin{tabular}{|l|c|l|l|l|c|}
\hline Cabeza y cuello & $\mathbf{n}(\%)$ & Tronco y miembros superiores & $\mathbf{n}(\%)$ & Pelvis y miembros inferiores & $\mathbf{n}(\%)$ \\
\hline Labios & $18(10,8)$ & Espalda & $5(3,0)$ & Sacra & $22(13,2)$ \\
\hline Fosa nasal & $17(10,2)$ & Tórax & $4(2,4)$ & Meato urinario & $15(9,0)$ \\
\hline Mejilla & $8(4,8)$ & Mano & $2(1,2)$ & Glúteo & $14(8,4)$ \\
\hline Pómulo & $7(4,2)$ & Abdomen & $1(0,6)$ & Ingle & $5(3,0)$ \\
\hline Encía & $6(3,6)$ & Brazo & $1(0,6)$ & Interglútea & $3(1,8)$ \\
\hline Pabellón auricular & $5(3,0)$ & Codo & $1(0,6)$ & Cadera & $1(0,6)$ \\
\hline Paladar & $5(3,0)$ & Mama & $1(0,6)$ & Trocánter & $1(0,6)$ \\
\hline Región subnasal & $3(1,8)$ & Cresta iliaca & $1(0,6)$ & Maléolo & $1(0,6)$ \\
\hline Tabique nasal & $3(1,8)$ & Radial derecha & $1(0,6)$ & Talón & $1(0,6)$ \\
\hline Comisura labial & $3(1,8)$ & Dedo & $1(0,6)$ & Testículo & $1(0,6)$ \\
\hline Occipital & $2(1,2)$ & Senos & $1(0,6)$ & Miembro inferior & $1(0,6)$ \\
\hline Cráneo & $1(0,6)$ & Esternotomía & $1(0,6)$ & Muslo & $1(0,6)$ \\
\hline Cuello & $1(0,6)$ & & & Muñón & $1(0,6)$ \\
\hline Frente & $1(0,6)$ & & & & $67(40,1)$ \\
\hline Total & $80(47,9)$ & Total & $20(12,0)$ & Total & \\
\hline
\end{tabular}

fue de categoría 2. Por ubicación anatómica, el 93,0\% se presentó en el meato urinario y el 7,0\% en el testículo. El 93,0\% de los pacientes tenía clasificación de riesgo alto.

La sonda nasogástrica ocasionó el 16,5\% de las LPD, y solo se dieron en adultos. La incidencia fue del 2,3\%. El 64,2\% se presentó en el servicio de hospitalización y el 35,8\% en la UCIA. El 64,2\% fue de categoría 2. El 100\% se presentó en la fosa nasal. El 71,4\% de los pacientes tenía clasificación de riesgo alto.

Las lesiones secundarias a la presencia de máscara de ventilación no invasiva fueron del 9,4\%; el $87,5 \%$ se dio en la UCICP. La incidencia en el paciente pediátrico fue del 7,7\%. El 75,0\% fue de categoría 1. De acuerdo con el sitio anatómico, el 37,5\% se presentó en el tabique nasal, el 25,0\% en fosa nasal, y el 12,5\% en pómulo, frente y pabellón auricular, respectivamente. El 100\% tenía clasificación de riesgo alto.

En cuanto al tiempo de aparición, las lesiones por tubo orotraqueal en el 50\% de los casos se presentaron a las 18 horas de colocado el dispositivo (rango intercuartílico [RIC] 5-48) en adultos; en los pacientes pediátricos fue de 24 horas (RIC 21-29). Con respecto a las lesiones por sonda vesical, la mediana fue de 72 horas (RIC 63-96). La mediana de sonda nasogástrica fue de 24 horas (RIC 17-36). La mediana de máscara de ventilación no invasiva en pacientes pediátricos fue de 72 horas (RIC 26-96).

\section{$Y$ LESIONES POR APOYO}

Se presentaron 48 lesiones por apoyo, que corresponde al 28,7\%. La incidencia fue del $0,8 \%$. El $87,5 \%$ se presentó en pacientes adultos. El $56,3 \%$ fue de categoría 1 y el 4,2\% de categoría 3. El servicio donde más se dieron fue en la UCIA $(41,6 \%)$, seguido por hospitalización de adultos $(39,5 \%)$ y en la UCICP $(6,2 \%)$. Los sitios más frecuentes fueron en la región sacra $(43,8 \%)$ y en la región glútea $(25,0 \%)$. En el 50,0\% de los casos, las lesiones aparecieron a los 5 días (RIC 3-10). El 62,5\% tenía clasificación de riesgo alto.

\section{Y LESIONES POR HUMEDAD}

Se encontraron 3 casos que corresponden al 1,8\%. Los pacientes eran mayores de 54 ańos. Dos se encontraban en la UCIA y uno en hospitalización. Las lesiones aparecieron entre las 24 y 48 horas. Dentro de los factores de riesgo se halló incontinencia urinaria y diarrea. Ninguna de las lesiones fue evitable.

No se presentaron lesiones por fricción.

\section{$\searrow$ MARSI}

Se produjeron 31 casos (18,6\%). En la UCICP se presentó en un 51,6\% y en la UCIA y hospitalización de adultos en un 16,2\%, respectivamente; en cirugía, 9,6\% y en pediatría y hemodinámica, 3,2\%. El 22,6\% se presentó en mejilla, el 19,4\% en pómulo, el 16,2\% en región subnasal y labios, y el $12,9 \%$ en ingle. Por clasificación, el $67,7 \%$ era de riesgo alto y el $45,2 \%$ fue MARSI evitable.

\section{У DISCUSIÓN}

La incidencia de lesiones por dependencia encontrada en este estudio es menor en comparación con otros estudios realizados en el mundo y en Colombia ${ }^{12-23}$, lo que puede deberse a la falta de cultura de reporte al ser consideradas como un daño colateral inherente a la atención de pacientes hospitalizados y no como un evento adverso que en la mayoría de los casos es prevenible ${ }^{21}$.

La categoría de las lesiones fue similar a la encontrada en una investigación en Burgos ${ }^{28}$. Esto puede deberse a que en ambas instituciones la identificación y/o análisis de los eventos fue realizado por personal de enfermería perteneciente al grupo de clínica de heridas, lo que facilitó la identificación en etapa temprana de este tipo de lesiones y su adecuado manejo.

Se encontró que la mayoría de los pacientes que presentaron lesiones por dependencia tenían riesgo alto según la escala de valoración, hallaz- 
go similar a lo reportado en otros estudios realizados en Bucaramanga y México ${ }^{29,30}$, lo que permite concluir que no es suficiente la aplicación de un instrumento de identificación del riesgo si no se adiciona acciones específicas de cuidado que prevengan cualquier tipo de lesión.

De acuerdo con el sitio anatómico específico, la lesión más frecuente se presentó a nivel sacro coincidiendo con varias investigaciones ${ }^{14,17,28}$; sin embargo, en este estudio, debido a que el mayor porcentaje de lesiones fueron ocasionadas por dispositivos médicos, la región con más lesiones es la cabeza y el cuello.

La incidencia global de lesiones por dispositivos médicos fue similar a la reportada por Willock et al. ${ }^{31}$; sin embargo, fue mayor que lo reportado por Black et al. ${ }^{23}$, lo cual se puede explicar porque esta institución tiene una especialidad cardiovascular, lo cual hace que el uso de dispositivo sea alto, aumentándose en pacientes en oxigenación por membrana extracorpórea (ECMO), asistencia ventricular y con cardiopatías congénitas complejas.

Los servicios en los cuales las lesiones por dispositivos fueron más frecuentes coincide con la revisión sistemática de J-E Torra ${ }^{21}$. Esto puede deberse a la complejidad de los pacientes, que por sus comorbilidades los hace más susceptibles a presentar cualquier tipo de lesión por dependencia, específicamente en las unidades de cuidados intensivos.

En el servicio de pediatría, la literatura especializada reporta que la máscara de ventilación mecánica no invasiva es uno de los dispositivos que genera la mayor incidencia de lesiones por presión ${ }^{23}$, hallazgo similar al encontrado en esta investigación.

En el paciente adulto, los dispositivos médicos que más generaron lesiones fueron el tubo orotraqueal y la sonda nasogástrica, resultados similares a los obtenidos por Coyers FM et al. ${ }^{24}$.
Con relación a la baja prevalencia de las LESCAH y la ausencia de LPF, es un fenómeno que puede ser atribuible al desconocimiento por parte del personal asistencial en la identificación de los diferentes tipos de lesiones por dependencia, lo que lleva a un subregistro de estas o a que sean todas catalogadas como LPP.

La investigación mostró que la mayoría de las lesiones fueron eventos adversos prevenibles, lo que evidencia un incumplimiento de las medidas establecidas según la clasificación del riesgo de cada paciente para prevenir las lesiones por dependencia.

\section{$\searrow$ CONCLUSIONES}

Es de gran importancia continuar haciendo estudios que brinden información sobre la incidencia de los diferentes tipos de lesiones por dependencia, ya que las medidas de prevención y los cuidados de estas varían de acuerdo con el mecanismo de producción.

Es prioritario la creación de instrumentos que sean válidos y confiables para la identificación del riesgo de cualquier tipo de LPD que permitan establecer acciones de prevención con miras a disminuir la incidencia de aquellas

\section{Limitaciones}

Estudio descriptivo con una muestra no aleatoria que no permite hacer inferencias.

\section{Conflicto de intereses}

Los autores declaran no tener ningún conflicto de intereses.

\section{У BIBLIOGRAFÍA}

1. Colombia. Ministerio de Salud y de la Protección Social. Herramientas para promover la estrategia de la seguridad del Paciente en el Sistema obligatorio de Garantía de Calidad de la atención en Salud. Bogotá: El Ministerio; 2007.

2. Comunidad Autónoma del País Vasco. Dirección de Asistencia Sanitaria. Coordinación de Programas de Salud Pública y Seguridad del Paciente. Estrategia de Seguridad del Paciente 2013-2016. Álava, Vitoria-Gasteiz: 2013

3. Agreda S, Javier J, Torra J, Posnett J, Verdú J, San Miguel L, et al. Una aproximación al impacto del costo económico del tratamiento de las úlceras por presión en España. Gerokomos. 2007;18(4):43-52.

4. Colombia. Ministerio de Salud y Protección Social. Prevención úlceras por presión. Paquetes instruccionales. Guía técnica "buenas prácticas para la seguridad del paciente en la atención en salud" Versión 2.0. 2015

5. García-Fernández FP, Soldevilla-Ágreda JJ, Pancorbo-Hidalgo PL, Verdú Soriano J, López-Casanova P, Rodríguez-Palma M. Clasificación - categorización de las lesiones relacionadas con la dependencia. Serie Documentos Técnicos GNEAUPP no II. Grupo Nacional para el Estudio y Asesoramiento en Úlceras por Presión y Heridas Crónicas. Logroño; 2014.

6. McNichol L, Lund C. Rosen T, Gray M. Medical adhesives and patient safety: state of the science: consensus statements for the assessment, prevention, and treatment of adhesive-related skin injuries. Orthop Nurs. 2013;32(5):267-81.

7. National Pressure Ulcer Advisory Panel, European Pressure Ulcer Advisory Panel and Pan Pacific Pressure Injury Alliance. Prevention and Treatment of Pressure Ulcers: Quick Reference Guide 2a ed. Australia; 2014

8. Colombia. Ministerio de Salud y Protección Social. Instituto Nacional de Vigilancia de Medicamentos y Alimentos INVIMA. ABC de dispositivos médicos. Bogotá; 2013.

9. Reger SI, Ranganathan VK, Sahgal V. Support surface interface pressure, microenvironment, and the prevalence of pressure ulcers: an analysis of the literature. Ostomy Wound Manage. 2007:53(10):50-8

10. García FP, Agreda JJ, Verdú J, Pancorbo PL. A new theoretical model for the development of pressure ulcers and other dependence-related lesions. J Nurs Scholarsh. 2014;46(1):28-38.
11. Lambert D. Prevention of incontinence-associated dermatitis in nursing home residents. Annals of Long-Term Care: Clinical Care and Aging. 2012;20(5):25-9.

12. Kottner J, Wilborn D, Dassen T, Lahmann N. The trend of pressure ulcer prevalence rates in German hospitals: results of seven cross-sectional studies. J Tissue Viability. 2009;18(2):36-46.

13. Vanderwee K, Clark M, Dealey C, Gunningberg L, Defloor T. Pressure ulcer prevalence in Europe: a pilot study. J Eval Clin Pract. 2007;13(2):227-35

14. Pancorbo PL, García P, Torra J, Verdú J, Soldevilla JJ. Epidemiología de las úlceras por presión en España en 2013: 4. Estudio Nacional de Prevalencia. Gerokomos. 2014:25(4):162-70.

15. Kelleher AD, Moorer A, Makic MF. Peer-to-peer nursing rounds and hospital-acquired pressure ulcer prevalence in a surgical intensive care unit: a quality improvement project. J Wound Ostomy Continence Nurs. 2012;39(2):152-7.

16. Rogenski NM, Kurcgant P. The incidence of pressure ulcers after the implementation of a prevention protocol. Rev Lat Am Enfermagem. 2012;20(2):333-9.

17. Gonzalez R, López W, Roa K. Epidemiología de lesiones de piel relacionadas con el cuidado: estudio de la prevalencia en Colombia. Revista Enfermagem Referência 2017 Oct-Dic; Série IV(15).

18. Tamayo C, Palacio C, Osorio C, Román J, Rodríguez L. Comportamiento de las úlceras por presión en los servicios de unidad de cuidados intensivos y unidad cuidados especiales en una clínica de alta complejidad de la ciudad de Medellín durante el primer semestre de 2014. CES Salud Pública. 2015;6:27-34.

19. Yepes D. Molina F León W. Pérez E. Incidencia y factores de riesgo en relación con las úlceras por presión en enfermos críticos. Med Intensiva. 2009;33:276-81.

20. Pérez G, Bosch A, Agustín C, Torra J. Prevalencia de lesiones por presión, incontinencia, dermatitis asociada a la incontinencia y factores de riesgo para el desarrollo de lesiones por presión en un hospital materno-infantil de tercer nivel. Gerokomos. 2018:29(2):83-91.

21. Torra JE. Incidencia de úlceras por presión en unidades de cuidados intensivos. Revisión sistemática con Meta-análisis. [Tesis Doctorall. Alicante: Departamento de Enfermería Comunitaria,
Medicina Preventiva y Salud Pública e Historia de la Ciencia de la Universidad de Alicante; 2016

22. Black JM, Cuddigan JE, Walko MA, Didier LA, Lander MJ, Kelpe MR. Medical device related pressure ulcers in hospitalized patients. Int Wound J 2010.7/5):358-65.

23. Kayser S, VanGilder C, Ayello E, Lachenbruch C. Prevalence and Analysis of Medical Device-Related Pressure Injuries: Results from the International Pressure Ulcer Prevalence Survey. Adv Skin Wound Care. 2018;31(6):276-85

24. Coyer FM, Stotts NA, Blackman VS. A prospective window into medical device related pressure ulcers in intensive care. Int Wound J. 2014:11:656-64

25. Mehta C, Ali M, Mehta Y, George JV, Singh MK. MDRPU -an uncommonly recognized common problem in ICU: A point prevalence study. J Tissue Viability. 2019;28 (1):35-9.

26. Roca-Biosca A, García-Fernández F, Chacón-Garcés S, Rubio-Rico L, Molina-Fernández M, Anguera-Saperas L, et al. Identificación y clasificación de las lesiones relacionadas con la dependencia: de la teoría a la práctica clínica. Gerokomos. 2015:26(2):58-62.

27. Konya C, Sanada H, Sugama J, Okuwa M, Kamatani Y, Nakagami G, Sakaki K. Skin injuries caused by medical adhesive tape in older people and associated factors. J Clin Nurs. 2010;19/910):1236-42

28. Real López L, Diez Estébanez ME, Serrano Hernantes M, de la Iglesia García E, Blasco Romero I, Capa Santamaría S, et al. Prevalencia de úlceras por presión y lesiones cutáneas asociadas a la humedad en el Hospital Universitario de Burgos. Gerokomos. 2017;28(2):103-8.

29. Valero-Cardenas H, Parra D, Rey-Gómez R, Camargo-Figuera F Prevalencia de úlcera por presión en una institución de Salud. Salud UIS. 2011;43(3):249-55.

30. Barrera J, Pedraza M. Pérez G. Hernández P, Reves J, Padilla M. Prevalencia de úlceras por presión en un hospital de tercer nivel en México DF. Gerokomos. 2016;27(4):176-81.

31. Willock J, Habiballah L, Long D, Palmer K, Anthony D. A comparison of the performance of the Braden $Q$ and the Glamorgan paediatric pressure ulcer risk assessment scales in general and intensive care paediatric and neonatal units. J Tissue Viability. 2016 May:25(2):119-26. 